\title{
ENTRE EL UNO Y EL CERO. EL ROL DEL DOCENTE Y EL ESTUDIANTE EN LA INVESTIGACIÓN-ACCIÓN EN INFORMÁTICA EDUCATIVA EN COLOMBIA
}

\author{
Widman Said Valbuena ${ }^{61}$
}

\section{RESUMEN}

El presente artículo plantea el rol y el lugar del docente y del estudiante en el contemporáneo interés de la investigación en informática educativa, además de la inclusión masiva de tecnologías de la información y la comunicación en los contextos escolares.

Desde un abordaje de la implementación y el desarrollo de tecnologías de la información y la comunicación para los contextos educativos como un fenómeno contemporáneo, se plantea la idea de una metodología de investigación que se ha decidido denominar IAIE o investigaciónacción en informática educativa como variante de la investigación-acción educativa (IAE), puesto que resalta y diferencia el rol del docente y del estudiante.

Se sostiene en la IAE que es el docente el responsable del cambio de su práctica. Sin embrago, el acto educativo del aula no es un acto individual, es por su naturaleza colectivo. Es decir, no es solo el docente el actor en este escenario, también lo son los estudiantes y según se sostiene en la IAE este método se orienta hacia el cambio de las condiciones de la realidad social desde la participación de los actores involucrados en el fenómeno. En ese sentido vale la pena preguntarse ¿Cuál sería el rol investigativo de los estudiantes en la transformación del fenómeno social educativo del cual hacen parte?, ¿La investigación acción educativa solo debe invitar al docente a participar en el proceso de construcción de conocimiento y transformación de la realidad educativa?

Palabras clave: Red iberoamericana de informática educativa (RIBIE), investigación acción educativa (IAE), investigación acción en informática educativa (IAEI).

\begin{abstract}
This article presents the role and place of teacher and student in the contemporary interest in educational computing research, plus the massive inclusion of information technology and communication in a school context.

From an approach to implementation and development of information technologies and communication in educational contexts as a contemporary phenomenon raises the idea of a research methodology that it's going to be called AREC or action research in educational

61 Investigador grupo Gestión Vital, Universidad Nacional Abierta y a Distancia UNAD. Editor revista de investigaciones UNAD. Magister en hábitat Universidad Nacional de Colombia. Candidato a especialista en Pedagogía del diseño, Universidad Nacional de Colombia.
\end{abstract}


computing as an alternative of educational action research (EAR), since it differences and highlights the role of teacher and student.

It rests on the EAR that the teacher is responsible for changing his/her practice. However, the classroom teaching act is not an individual act, it's by nature collective. That is, the teacher is not only the actor in this scenario, so are the students and it is argued in the EAR that this method is oriented towards the changing conditions of social reality from the participation of actors involved in the phenomenon. In this regard it is worth asking, What would be the students' research role in transformation of the educational social phenomenon of which are part of? Should Educational action research invite only the teachers to participate in the process of knowledge construction and transformation of the educational reality?

Key words: Ibero-American Network of Educational Informatics (IANEI), educational action research (EAR), action research in educational computing (AREC).

Recibido: 16 de septiembre de 2009

Aceptado: 23 de octubre de 2009

\section{El lenguaje binario de la maquina en la retórica de la escuela}

Desde sus albores la informática ha basado su lenguaje en el sistema numérico denominado binario donde es posible escribir cualquier cifra usando únicamente dos dígitos, el 1 (uno) y el cero (0) cuyas combinaciones han alcanzado gran nivel de complejidad a partir de dos niveles de voltaje, encendido (1) y apagado (0).

Algunos productos derivados de la complejidad en la combinación de estos dígitos se han introducido en el contexto educativo en forma de software acompañados, por su puesto, de las máquinas informáticas que caracterizan nuestra actual forma de vida. Computadores, redes y otros dispositivos que procesan los dígitos han sido introducidos en las aulas, bajo diferentes eslóganes que promueven el mejoramiento de la educación aludiendo a las indudables características comunicativas que poseen dichas máquinas y su lenguaje binario.

El desarrollo del uno y el cero ha derivado, entre otras, en una corriente de desarrollo tecnológico denominado informática educativa, la cual incluye el diseño instruccional, el desarrollo de software para sector educativo y en paralelo el desarrollo de hardware. Este nuevo panorama en la educación ha requerido entonces de procesos de investigación orientados desde sus inicios, a dos corrientes; la primera enfocada hacia el diseño de programas denominados software educativo o didáctico. Si este viene acompañado de un diseño metodológico de incorporación a los procesos educativos se denomina, en conjunto, diseño instruccional. Por otro lado, está la descripción, y en algunos casos la explicación, de la inserción de estos nuevos dispositivos en las aulas, tratando de medir o mostrar el impacto que tienen las tecnologías de la información y la comunicación en los procesos que allí se desarrollan. 
Para dar cuenta de los procesos de la investigación en contextos escolares se han implementado estrategias de visibilidad de los productos obtenidos. Como ejemplo tenemos las publicaciones en revistas y eventos para socializar las experiencias investigativas, congresos, seminarios, encuentros de investigadores, foros. Uno de los más recientes y si se quiere con mayor tradición en Colombia es el organizado por la red Iberoamericana de Informática Educativa nodo Colombia (en adelante RIBECOL.) En este, como en los demás eventos de la misma naturaleza, se presentan experiencias que tienen que ver con cualquiera de las corrientes de investigación e implementación antes mencionadas, donde se amalgaman el lenguaje de la máquina y los discursos propios de los entornos educativos, convirtiendo estos eventos en vitrinas para visibilizar el avance de la investigación en informática educativa en Colombia y para establecer puentes y redes entre grupos de investigación afines consolidando así la gestión del conocimiento.

\section{La investigación acción educativa}

En los últimos tiempos se ha insistido, por parte de algunos académicos cercanos a los procesos educativos, en la importancia que tiene la investigación del complejo mundo de la educación donde existen diferentes abordajes, e inclusive diferentes objetos de investigación dependiendo de la disciplina que decida acercarse a los problemas que este campo plantea y que conforman un dominio académico denominado ciencias de la educación derivado de las ciencias sociales, entre ellas la psicología, la sociología, la pedagogía y la antropología (Restrepo. 2004). Estas disciplinas, cuando centran su atención en el mundo de la educación, llevan consigo también los métodos afinados desde sus propios campos disciplinares, los cuales dependen, además, del paradigma en que se mueva el investigador.

Es así como se encuentran estudios en educación que reflejan diseños metodológicos propios del positivismo clásico de las ciencias naturales, donde términos como "experimental", "cuasi-experimental", "grupo control", "grupo experimental” y otros, muy propios todos del discurso positivista de la ciencia que busca, por lo general, corroborar hipótesis en un modelo cuantitativo por excelencia. Pero también se encuentran en el argot científico tendencias más cualitativas con enfoques exploratorios, hermenéuticos, socio-críticos donde no es imprescindible la hipótesis si no la construcción del conocimiento desde el acercamiento a éste en su estado más natural.

La investigación en informática educativa no está ausente de estos modos de construcción de conocimiento ya sea en el desarrollo de dispositivos tecnológicos con fines educativos o en la indagación por el uso de estos en los entornos escolares. No existe una correlación directa entre las dos corrientes y las dos formas más generales de clasificar el proceder científico en la educación, se pueden encontrar investigaciones que apunten al desarrollo tecnológico centradas en un enfoque positivista, pero con aportes de corte cualitativo, del mismo modo se encuentran acercamientos cualitativos al aula para mira los efectos que producen los artefactos en el desarrollo de las dinámicas escolares con diseños experimentales de orden cuantitativo. 
Una perspectiva relativamente contemporánea es la incorporación de la investigación acción participativa o IAP en la educación con el fin, ya no solo de conocer el complejo fenómeno de la educación en el aula, si no también, desde una perceptiva socio-crítica, de transformar el fenómeno educativo que se presenta diariamente en el aula buscado de este modo cualificar los procesos de enseñanza y aprendizaje. Esta corriente de la IAP se conoce en la actualidad como investigación-acción educativa o IAE.

\section{Dos corrientes de investigación en informática educativa: Un nuevo camino}

Como se anotó con anterioridad existen dos caminos originales en el estudio de la informática educativa, uno es el que centra sus acciones en el desarrollo de dispositivos (hardware y software) y el otro que intenta auscultar en el impacto que tiene el uso de estos dispositivos en las dinámicas del aula.

En el primero se han hecho importantes desarrollos de software, con diferentes matices, muchos de estos amarrados a modelos pedagógicos tratando de emular procesos cognitivos y elementos propios de cada modelo. En la historia del diseño instruccional en relación con las tics se puede ver en detalle cómo a cada nuevo desarrollo le corresponde un nuevo periodo en la forma en que se concibe el aprendizaje (Polo, 2001).

En el otro sentido, no se centra el estudio en el desarrollo tecnológico con fines educativos, si no en tratar de entender cómo los actores escolares (docentes y estudiantes) interactúan en un entorno intervenido con tics. En esta perspectiva se mantiene constantemente latente la crítica de la pertinencia de estos dispositivos en el aula, tratando de extraer de las características de los artefactos aquellos elementos que puedan intervenir positivamente en los procesos de aprendizaje en el aula.

Un buen ejemplo de esta perspectiva fue el presentado por el grupo de investigación DIDACTEC (didáctica de la tecnología) en el $10^{\circ}$ Foro de investigadores en informática educativa organizado por RIBIECOL, denominado "Computadores portátiles e internet en la escuela: relato de una experiencia". ${ }^{62}$ En este proyecto se aborda el impacto que puede tener el uso de estas tecnologías en términos de aprendizaje, su planteamiento es altamente cualitativo, pero posee un diseño metodológico de grupo control y grupo experimental, lo que le permitió a los investigadores comparar los procesos de aprendizaje que se llevaron a cabo entre estos grupos.

Como se puede ver, existe en la actualidad una interesante combinación de métodos en la investigación educativa referida a la inserción de tics. Del mismo modo se encuentran ejemplos interesantes donde las dos vertientes de este campo de investigación han encontrado puntos de convergencia. Un buen ejemplo del encuentro entre líneas de investigación también se

62 Ponencia presentada en el $10^{\circ}$ Foro de investigadores en informática educativa; RIBIECOL 2009, la cual obtuvo el segundo puesto en el marco del premio a mejor investigación en este campo. Esta ponencia fue presentada por el grupo de investigación didáctica de la tecnología - DIDACTEC - Universidad Distrital Francisco José de caldas, Facultad de Ciencias y Educación. 
presentó en el Foro RIBIECOL 2009. En el proyecto denominado Cupi2 $2^{63}$ se comenta por parte de sus autores que:

"el proyecto se orientó hacia el diseño y desarrollo de recursos informáticos, junto con una propuesta pedagógica innovadora y un sólido marco conceptual, para abordar la problemática del desarrollo de competencias en programación de los estudiantes de ingeniería". (Entrevista equipo proyecto Cupi2).

Lo anterior da cuenta de una interesante dinámica de retroalimentación en la lógica interna del proceso metodológico. Según los autores, en este equipo existen investigadores y desarrolladores de software, observadores de la implementación en el aula, generalmente pedagogos o expertos en temáticas educativas y los docentes del departamento de ingeniería que por medio de convocatoria quisieron participar en el proyecto. El trabajo realizado por los diseñadores e ingenieros es puesto a prueba en las aulas, esto último lo hacen los docentes previa formación por parte del equipo de investigación, una vez puesto a prueba el docente es acompañado por un observador quien registra la implementación en el aula. Su registro tiene dos caminos, el primero es la retroalimentación con el docente y el segundo es el envío a los investigadores para que se realice todo el trabajo de análisis, de donde salen elementos que les permite a los desarrolladores modificar sus productos, para comenzar de nuevo el ciclo.

Este ejemplo admite materializar, entre otras cosas, la idea del aula como laboratorio, según Bernardo Restrepo el docente debe llevar "el currículo al laboratorio del aula como una hipótesis que tiene que someter a prueba y a las adaptaciones que este experimento sugiera." (Restrepo, 2004), y si dentro del currículo se encuentran las tecnologías de información y comunicación estas son, por su puesto, parte del experimento.

\section{IAIE, investigación acción en informática educativa}

Así como la IAE es una variante de la IAP, se puede pensar que la investigación acción en informática educativa (IAIE) es igualmente una variante de estas. Existen experiencias significativas de aplicación de IAE donde al intervenir el fenómeno educativo se logra básicamente transformar las prácticas docentes. (Ibíd., 2004).

Estas experiencias se caracterizan por involucrar de forma activa al docente en la comprensión de su propia práctica y en su posterior transformación, lo cual es coherente con lo que busca este enfoque socio-crítico de las Ciencias Sociales. En ese sentido se espera que si el fenómeno escolar es intervenido con tics estas sean parte del objeto de estudio y así como se pudo observar en el proyecto Cupi2, estas sean rediseñadas gracias a la información que se obtiene de su implementación.

63 Este proyecto fue presentado por el Jorge Villalobos y Nadya Calderón de la universidad de los Andes, el cual obtuvo el primer puesto en el marco del premio RIBIECOL 2009 a mejor investigación en este campo. 
Algo que caracteriza los enfoques IAP, además de la transformación de la realidad social, es que para que esa transformación sea un hecho se involucran los actores sociales como investigadores de su propia realidad, se deconstruye y reconstruye ésta según como lo consideren los mismos actores en conjunto con los investigadores externos. La educación es ante todo un fenómeno social ya que adquiere su sentido únicamente en colectivo. Como fenómeno social participan diferentes actores, entre los que se cuentan directivos, docentes, estudiantes, familias, entre otros. En particular en el aula, se establecen relaciones entre docente y estudiante para materializar la enseñanza y el aprendizaje. Desde un abordaje estricto del la IAE, en el estudio del fenómeno del aula deberían participar activamente no solo los docentes si no también los estudiantes, más si lo que se busca es que estos últimos sean activos participantes de sus procesos de aprendizaje. En ese sentido ¿qué tendrían que decir los estudiantes del uso de tics en el aula? ¿qué argüirían estos a favor o en contra del método de enseñanza?, ¿cómo considerarían estos mismos debería ser su rol dentro del aula?

En su estudio Marc Jané, hace un interesante experimento centrado sobre todo en la evaluación. Diferencia evaluación sumativa de evaluación formativa y opta por experimentar con ésta última (Jané, 2004). En su estudio involucra a los estudiantes de arquitectura de la Universidad de los Andes para que participen en el diseño de instrumentos de evaluación plateando en conjunto los criterios a tener en cuenta en el proceso de aprendizaje y luego desarrollando lo que en su trabajo opta por denominar evaluación colaborativa entre pares, donde la dinámica permite que, a la luz de los criterios planteados en colectivo, los estudiantes se aventuren a opinar críticamente sobre sus propios trabajos y sobre los trabajos de sus compañeros.

En el caso de la IAEI se esperaría que los estudiantes participaran también en los criterios de evaluación de las competencias que se desarrollan o se inhiben por el uso de las tics en el aula (Valbuena, 2009), que, de ser posible, puedan dar elementos para el rediseño de dispositivos tecnológicos, que están pensados para incluirlos en las agendas de trabajo escolar. Como observa Fabio Jurado parafraseando a Voloshinov, "la intersubjetividad es inherente a la investigación participativa por la gama de significados que se cruzan según sean las percepciones de los participantes en la investigación -en nuestro caso el profesor y los estudiantes-. Los participantes dirimen, se animan con las contradicciones y cada error es una potencialidad para comprender el fenómeno observado. Pedagogía y hermenéutica se conjuntan con la investigación.”(Jurado, s.f.) (cf. Voloshinov, 1992).

\section{El docente en la IAIE}

La implementación de tecnología de la información y la comunicación puede traer consigo beneficios implícitos en los proceso de aprendizaje, pero también tensiones muy altas ya que su uso no es común en las aulas todavía. El docente en su práctica no se encuentra mimetizado con estas tecnologías y puede ocasionar, como es normal, conflicto con las formas tradicionales de desarrollar una clase (Valbuena, 2009). 
El docente es un actor fundamental en los enfoques de intervención cualitativa ya que se busca transformar la realidad que se vive en las aulas. Sin embargo generalmente se ha orientado la IAE a la transformación de la práctica docente, lo cual centra el estudio solo en una parte del fenómeno. Esto no quiere decir que no se hayan tenido experiencias exitosas con este tipo de abordaje de la IAE, ya lo vimos en el proceso desarrollado en el proyecto Cupi2, donde el docente asistido por el grupo de investigación recibe contantemente retroalimentación de la implementación de tics en su aula, lo que lleva no solo a la transformación de su práctica a partir de la reflexión crítica de ésta, (Shön, 1992), sino también al replanteamiento de las características de la tecnologías que allí se están implementando con el fin de ajustarlas a la práctica en una lógica retroactiva, propia de este tipo de investigación.

El docente adquiere entonces un rol protagónico en este tipo de experiencias investigativas. Para el caso del proyecto ganador del premio RIBIECOL 2009, este rol adquiere unos matices interesantes.

\footnotetext{
"los docentes inicialmente son invitados a participar en el proyecto por medio de una convocatoria que hace la universidad, su papel en el proyecto es seguir siendo docentes, no actúan como investigadores de su práctica, ya que existen otros roles en el proceso...

...existen los observadores, quienes son los que toman registro de lo que ocurre en el aula y luego comparten sus observaciones con el docente, esto permite dos cosas, por un lado obtener información de la clase por parte del docente lo que nutre el registro y segundo permite al docente extraer algunos elementos en compañía del observador, para modificar la siguiente clase." (Entrevista equipo proyecto Cupi2).
}

El docente en esta experiencia sigue siendo docente, solo que es un docente consiente de estar en un proceso de investigación. Argumenta Jorge Villalobos director del proyecto, que se pensó de esta forma para evitar que el docente se concentrara en una tipo de observación rigurosa que no es, por lo general, un proceso natural en las clases de ingeniería y que además es una competencia investigativa que muchos docentes aun no han desarrollado. Por el contrario, esta variante ha permitido que el docente se concentre en su clase, la cual es reajustada en cada sesión gracias a la retroalimentación que comparte con el observador, el docente al estar haciendo su trabajo pero de una forma más consiente identifica elementos importantes para replantear o re-direccionar la clase con intervención de tics.

De acuerdo con el propósito del proyecto Cupi2, es interesante pensar en una variante de la IAE donde la participación de los actores sea diferenciada desde sus potencialidades, y en el caso en que los docentes no muestren competencias investigativas, sean asistidos por otros profesionales, compartiendo la experiencia docente y la experiencia investigativa, y así como el docente permite que un extraño ingrese a su aula para observar su clase, el investigador o el observador permite que el docente "ingrese" a su registro para compartir el contenido registrado. Es una relación dialógica donde se comparte información para construcción de nuevo conocimiento que a su vez permita la transformación de lo que se está estudiando. 


\section{El estudiante en la IAIE}

Se sostiene en la IAE que es el docente el responsable del cambio de su práctica. Sin embargo, el acto educativo del aula no es un acto individual, es por su naturaleza colectivo, es decir, no es solo el docente el actor en este escenario, también lo son los estudiantes. Citando nuevamente a Fabio Jurado diríamos que compartir una clase es un fenómeno intersubjetivo donde afloran visiones de mundo y valores e intereses entre otros, lo que a mi juicio complejiza la denominada clase.

Involucrar a los estudiantes tiene un alto potencial en la transformación de la realidad social, como se pudo observar en el experimento que planteó Marc Jané, incluir a sus estudiantes en el diseño de instrumentos de evaluación definiendo en conjunto los juicios valorativos del trabajo de la clase, permitió a los estudiantes adelantar procesos metacognitivos para darse cuenta de la forma en que cada uno estaba aprendiendo, donde un buen número de estos estudiantes modificó la percepción que tenían de la evaluación desde un enfoque meramente cuantitativo a un enfoque mucho más cualitativo, en términos de Jané, los estudiantes dejaron de pensar que la evaluación era un problema de contabilidad para darse cuenta que era una forma de aprender (Jané, 2004).

En ese sentido si en realidad se quiere implementar una metodología de investigación cualitativa en la incorporación de tecnologías de la información y la comunicación en el aula, de modo que se pretenda una transformación de esta realidad social con miras a su cualificación, se debe tener presente que en el aula los estudiantes son participantes, actores en un escenario complejo que tiene muchas cosas que aportar para generar una autonomía en el proceso desarrollando las competencias que le permitirán desempeñarse cuando ya no hagan parte de ese contexto y cuando ya su profesor(a) no se encuentre para orientarlo.

Cuando se decide introducir el uno y el cero al aula se incrementa la complejidad del fenómeno, porque se entremezclan dos complejidades, la que trae la máquina con la que caracteriza el contexto educativo, lo cual requiere de atención constante para sortear, por medio de la observación, la reflexión de y en la práctica, el análisis, la explicación y la participación de todos sus actores y autores, los avatares de una complejidad multidimensional y que, además, se requiere continuamente estar transformando.

\section{REFERENCIAS BIBLIOGRÁFICAS}

BRICEÑO SERGIO, MOLINA RUTH \& QUINTANA ANTONIO. (2009). Computadores portátiles e internet en la escuela: relato de una experiencia. Universidad Distrital Francisco José de Calas. Ponencia segundo puesto del premio a mejor investigación en el marco del $10^{\circ}$ foro de investigadores en informática educativa, ROBIECOL 2009.

JANÉ, MARC. (2004), Evaluación y aprendizaje de la arquitectura, efectos de la autoevaluación, de la evaluación por pares y la evaluación por colaboración, en el aprendizaje 
del diseño arquitectónico. Informe final de tesis, Maestría en educación. Universidad de los Andes. CIFE, centro de investigación y formación en educación.

JURADO V, FABIO. Investigación, pedagogía y evaluación en el aula universitaria. Manuscrito sin fechar. Universidad nacional de Colombia.

POLO, MARÍA (2001). El diseño instruccional y las tecnologías de la información y la comunicación. Docencia Universitaria, Vol. II, N 2. 2001.

RESTREPO G, BERNARDO. (2004) La investigación-acción educativa y la construcción de saber pedagógico. En revista Educación y educadores, volumen 7, Universidad de la sabana. 2004.

VALBUENA B. WIDMAN, (2009), Reset y F5, ¿Son las tics una necesidad en la escuela actual? En Revista de investigaciones UNAD. Vol. 8 Núm. 1. Universidad Nacional Abierta y a distancia. UNAD. 2009.

VILLALOBOS, JORGE \& CALDERÓN NADYA. (2009). Proyecto Cupi2: Un Enfoque Multidimensional Frente Al Problema De Enseñar Y Aprender A Programar. Universidad de los Andes. Ponencia primer puesto del premio a mejor investigación en el marco del $10^{\circ}$ foro de investigadores en informática educativa, ROBIECOL 2009. 